\title{
A NEW HYBRID ALGORITHM FOR THE MODIFIED BARRIER FUNCTION FORM
}

\author{
ABBAS Y. AL-BAYATI \\ Prof. of numerical optimization \\ ASEEL MOAYAD QASIM
}

Department of mathematics

College Of Computer Sciences and Mathematics

Mosul University

Received

06 / 09 / 2007
Accepted

03 / 12 / 2007

\section{الملغص}

قمنا في هذا البهث بطوير صيغة جيدة أكثر ملائمة لدالة Barrier مع لستحداث خوارزمية هجينة جدية والمتضمنة طريقة الانحدار المتدرج مع المتري المتغير وبلستعمل خط بحث تلم في همل الأمثلية غير الخطية والمقية.

\begin{abstract}
In this paper, we have developed a new formula which is more suitable to the Barrier function and we have also investigated a new hybrid algorithm of SD and VM formulas with exact line search in the field of non-linear constrained optimization.
\end{abstract}




\section{INTRODUCTION:}

\subsection{A STANDARD BARRIER FUNCTON:}

This approach is suitable for inequality constraints only. the function $\varphi(x, \rho)$ are of the form $\varphi(x, \rho)=f(x)+\rho_{k} \sum_{i=1}^{m} \varphi\left\{c_{i}(x)\right\}$ denote $\varphi_{i}=\varphi\left\{c_{i}(x)\right\}$ where $\rho_{k}$ : Is appositive scalar.

$f(x)$ : Is the objective function .

$\mathrm{m}$ : number of constraints.

$\varphi$ : Is the Barrier function .

There are many types of Barrier methods , see [ 1 , 4 , 3 ] such as :

$$
\begin{aligned}
& B(x)=\sum_{j=1}^{m} \frac{1}{c j(x)} \quad, \quad \ldots . \\
& B(x)=\sum_{j=1}^{m} \frac{1}{c j(x)} \varepsilon \quad, \varepsilon>0 \\
& B(x)=-\sum_{j=1}^{m} \ln (c j(x))
\end{aligned}
$$

Carrol in 1961 developed the inverse barrier function and in fact this idea further analytic and implemented by Fiaco and Mc. Cormic in 1964 and its known as the SUMT - method where SUMT stand for (sequential unconstrained minimization technique) the whole problem is now :

$$
\min \varphi(x, \rho)=f(x)+\rho_{k} \sum_{i=1}^{m} 1 / c_{i}(x)
$$

\subsection{A STANDARD PENALTY FUNCTON:}

Barrier function is not suitable for equality constrained because there is no way of ensuring that $\mathrm{x}$ satisfies all the constraints as equalities. Penalty function method follow similar procedure but the modification to the objective function comes into play when the constraints are violated and increases as the violation increases.

The Penalty function is of the form

$$
\varphi(x, \rho)=f(x)+\frac{1}{\rho} \sum_{i=1}^{m} \varphi\left\{c_{i}(x)\right\}
$$


if $\varphi_{i}=\varphi\left\{c_{i}(x)\right\}$

Then either $\varphi_{i}=c^{2}{ }_{i}(x)$

this is usually used for the equality constraints or $\varphi_{i}=\left[\min \left\{0, c_{i}(x)\right\}\right]^{2}$

this is used for the inequality constraints.

The function $P(x): R^{n} \longrightarrow R$ is called Penalty function to the equation

$\min f(x)$

$$
\begin{aligned}
& \text { s.t. } \quad g_{j}(x) \leq 0 \quad j=1, \ldots m 1 \\
& h_{k}(x)=0 \quad k=1, \ldots m 2 \\
& x_{i}^{l} \leq x_{i} \leq x_{i}^{u} \quad i=1,2, \ldots n \quad, l, u \text { : upper and lower bounds }
\end{aligned}
$$

which is satisfies [8] :

$$
\begin{aligned}
& P(x)=0 \text { if } h(x)=0, c(x) \geq 0 \ldots \\
& P(x)>0 \text { if } h(x) \neq 0 \quad \text { or } c(x)<0
\end{aligned}
$$

The general outlines of any algorithm used to solve such problems are as follows [7]:

1. Minimize objective as unconstrained function.

2. Provide penalty to limit constraint violations.

3. Magnitude of penalty varies through the optimization.

4. Called sequential unconstrained minimization technique (SUMT).

5. create pseudo - objective $\phi\left(x, r_{p}\right)=f(x)+r_{p} P(x)$ Such that:

$f(x)=$ Original objective function.

$P(x)=$ Imposed Penalty function.

$r_{p}=$ Scalar multiplier to determine penalty magnitude .

$p=$ Unconstrained minimization number.

and there is four basic algorithms to solve such problem :

1. Exterior penalty function method.

2. Interior penalty function method.

3. Log penalty function method.

4. Extended interior penalty function method.

The effect of Penalty function is to create a local minimum of unconstrained problem near $x^{*}$. 
Penalty function adds a penalty for infeasibility, barrier function adds a term that prevents iterates from becoming infeasible.

\subsection{AMODIFIED APPROACH TO THE BARRIER AND PENALTY FUNCTIONS [9]:}

Since the Barrier methods is a strictly methods,

$$
P\left(x, r_{p}\right)=f(x)+r B(x)
$$

Where $f$ is a maximizing function and $\boldsymbol{B}$ is a barrier function. Consider the following:

$$
\begin{aligned}
& \min \quad f(x) \\
& \text { s.t. } \quad g_{i}(x) \leq b_{i} \\
& x_{i} \geq 0 \\
& B(x)=\sum_{i=1}^{m} \frac{1}{b_{i}-g_{i}(x)}+\sum_{j=1}^{n} \frac{1}{x_{j}}
\end{aligned}
$$

Remarks:

1. if $b_{i} \longrightarrow g_{i}(x)$ then $B(x) \longrightarrow \infty$.

2. SUMT methods applied to the non-linear problems.

3. One of the most difficult tasks when using SUMT is the choice of the initial penalty parameters.

This is because the initial choice of penalty parameters is often a problem dependent and almost always has a major effect on overall optimization efficiency.

Therefore, we try to pick starting values for penalty parameters which will ensure a well-posed, efficient optimization task.

\subsection{THE (SUMT) ALGORITHM:}

The idea of SUMT methods is to solve the unconstrained $\min p(x, r)$ Over a sequences of times. Each sequence reduces $r$ i.e allows us to move closer to boundary. Usually go until some error tolerance is reached.

If

$x_{1}=\max \left(P(x, r), x^{*} \max f(x)\right)$ 
then

$f\left(x_{1}\right) \leq f\left(x^{*}\right) \leq f\left(x_{1}\right)+r \cdot P\left(x_{1}\right)$

make $r_{\text {new }}=\vartheta . r \quad$, and let $\vartheta=0.1$ When the stooping criteria is $r \leq \varepsilon$

\subsection{OUTLINES OF SUMT ALGORITHM :}

1. let $\mathrm{x}=$ initial solution (not on boundary), $\mathrm{k}=1, \theta=0.01, \mathrm{r}=1$.

2. at k-th iteraton ,

$x_{k-1}=$ Starting point, we can use multivariate unconstrained technique Such that gradient search method to find the local maximum / minimum of

$p(x, r)=f(x)-r \cdot\left[\sum_{i=1}^{m} \frac{1}{b_{i}-g_{i}(x)}+\sum_{j=1}^{n} \frac{1}{x_{j}}\right]$

3. Stop if $\left|x_{k}-x_{k-1}\right| \leq \varepsilon$

4. Else $\mathrm{k}=\mathrm{k}+1$ and $r=r . \theta$ go to step 2 .

\section{EXTENDED INTERIOR PENALTY FUNCTION:}

Incorporate the best features of the exterior and interior methods so that a penalty function is continuous every where and provides a sequence of improving feasible suboptimum. With $g_{0}$ such that [5].

$$
g_{0}=-c\left(r_{p}\right)^{a} \text { where } \frac{1}{3} \leq a \leq \frac{1}{2}, \quad c=0.2 .
$$

\subsection{LINEAR EXTENDED PENALTY FUNCTION:}

The penalty function is used for inequality constraints as follows:

$P(x)=\sum_{j=1}^{m} g_{j}(x)$

if $g_{j}(x) \leq g_{0}$ then $p_{j}(x)=\frac{-1}{g_{j}(x)}$

if $g_{j}(x)>g_{0}$ then $p_{j}(x)=-\frac{2 g_{0}-g_{j}(x)}{g_{0}^{2}}$

and here we can define $g_{0}$ as : 
$g_{0}=-c\left(r_{p}\right)^{a}$

Which is a small negative number, $\mathrm{C}$ is a constant determined at the beginning as follows:

Choose $g_{0}$ in the range $-0.3 \leq g_{0} \leq-0.1$ then chose $r_{p}$ such that:

$r_{p}=|z| / \sum_{j=1}^{m} P_{j}(x)$

then

$c=-g_{0} /\left(r_{p}\right)^{a}$

\subsection{QUADRATIC PENALTY FUNCTON:}

Create a quadratic extended penalty function which has continuous second derivatives at $g_{j}(x)=g_{0}$ and

$$
\begin{gathered}
p_{j}(x)=\frac{-1}{g_{j}(x)} \quad, \quad g_{j}(x) \leq g_{0} \\
p_{j}(x)=-\frac{1}{g_{0}}\left\{\left[\frac{g_{j}(x)}{g_{0}}\right]^{2}-3\left[\frac{g_{j}(x)}{g_{0}}\right]+3\right\}, g_{j}(x)>g_{0}
\end{gathered}
$$

The quadratic extended penalty function may be more useful if a second-order method is used for unconstrained sub problems.

However, the degree of nonlinearity of $\Phi$ is increased, when incorporating the extended penalty function into an optimization program we should provide several options and experiment to see which is more efficient for the class of problems we are solving.

\section{A NEW GENERALIZED FORM TO THE DIFFERENCE BETWEEN THE CONSTRANED}

By taking the differences between the constrained in the tow cases when $g_{j}(x) \leq g_{0}$ and $g_{j}(x)>g_{0}$ and the extended penalty is linear we note that : 


$$
\begin{aligned}
& \frac{-2 g_{0}+g_{j}}{g_{0}^{2}}+\frac{1}{g_{j}} \\
= & \frac{g_{j}^{2}-2 g_{0} g_{j}+g_{0}^{2}}{g_{0}^{2} g_{j}} \\
= & \frac{-2 g_{0} g_{j}+g_{j}^{2}+g_{0}^{2}}{g_{0}^{2} g_{j}} \\
= & \frac{\left(g_{j}-g_{0}\right)^{2}}{g_{0}^{2} g_{j}}, \ldots . .
\end{aligned}
$$

and in the case when extended penalty is quadratic:

The formula of the constrained when $g_{j}(x)>g_{0}$ is the equation (21), and when we take the difference between the constraint in the two cases as the previous method, we note :

$$
\begin{aligned}
& -\frac{1}{g_{0}}\left\{\left[\frac{g_{j}}{g_{0}}\right]^{2}-3\left[\frac{g_{j}}{g_{0}}\right]+3\right\}+\frac{1}{g_{j}} \\
& =-\frac{g_{j}^{2}}{g_{0}^{3}}+3 \frac{g_{j}}{g_{0}}-\frac{3}{g_{0}}+\frac{1}{g_{j}} \\
& =\frac{-g_{j}^{3}+3 g_{j}^{2} g_{0}-3 g_{j} g_{0}^{2}+g_{0}^{3}}{g_{0}^{3} g_{j}} \\
& =-\frac{\left(g_{j}-g_{0}\right)^{3}}{g_{0}^{3} g_{j}} \ldots \ldots . . . \ldots . . . . .
\end{aligned}
$$

We note from the two previous cases the difference between the constraint when $n=1$ is as the equation (22) and when $n=2$ is as the equation (23), and from this we can conclude that the difference when $\mathrm{n}=3$ to be as the form :

$$
\frac{\left(g_{j}-g_{0}\right)^{4}}{g_{0}^{4} g_{j}}
$$

By substituting this relation in the followed method (by taking the difference between the formula) we can conclude the formulae when $g_{j}(x)>g_{0}$ and $\mathrm{n}=3$ to be in the following form:

$$
\frac{\left(g_{j}-g_{0}\right)^{4}}{g_{0}^{4} g_{j}} \text { cubic }+\frac{1}{g_{j}}=
$$




$$
\begin{aligned}
& \text { cubic }=\frac{g_{j}^{4}-4 g_{j}^{3} g_{0}+6 g_{j}^{2} g_{0}^{2}-4 g_{j} g_{0}^{3}+g_{0}^{4}}{g_{0}^{4} g_{j}}-\frac{1}{g_{j}} \\
& =\frac{g_{j}^{4}-4 g_{j}^{3} g_{0}+6 g_{j}^{2} g_{0}^{2}-4 g_{j} g_{0}^{3}+g_{0}^{4}-g_{0}^{4}}{g_{0}^{4} g_{j}} \\
& =\frac{g_{j}^{3}}{g_{0}^{4}}-4 \frac{g_{j}^{2}}{g_{0}^{3}}+6 \frac{g_{j}}{g_{0}^{2}}-4 \frac{1}{g_{0}} \\
& =\frac{1}{g_{0}}\left\{\left[\frac{g_{j}(x)}{g_{0}}\right]^{3}-4\left[\frac{g_{j}(x)}{g_{0}}\right]^{2}+6\left[\frac{g_{j}}{g_{0}}\right]-4\right\}, \ldots \ldots .
\end{aligned}
$$

and when $\mathrm{n}=4$ and in the similar way, the general form to the difference is equal to :

$$
-\frac{\left(g_{j}-g_{0}\right)^{5}}{g_{0}^{5} g_{j}}
$$

then the general form to the constrained when $g_{j}(x)>g_{0}$ according the mathematical induction is as follows :

1. when $n$ is odd:

$$
\frac{\left(g_{j}-g_{0}\right)^{n+1}}{g_{0}^{n+1} g_{j}},
$$

2. when $n$ is even:

$-\frac{\left(g_{j}-g_{0}\right)^{n+1}}{g_{0}^{n+1} g_{j}}$,

\subsection{A NEW GENERALIZED FORM OF THE CONSTRAINED FUNCTION WHEN $\boldsymbol{g}_{j}(\boldsymbol{x})>\boldsymbol{g}_{\mathbf{0}}$}

From our notation to the equations (22) ,(23), (24) , (26) we can conclude that the general form to the constrained when $g_{j}(x)>g_{0}$ to be in the following way: 
1. when $n$ is odd

$$
\frac{1}{g_{0}}\left\{\left[\frac{g_{j}(x)}{g_{0}}\right]^{n}-{ }_{1}^{n+1}\left[\frac{g_{j}(x)}{g_{0}}\right]^{n-1}+{ }_{2}^{n+1}\left[\frac{g_{j}}{g_{0}}\right]^{n-2}+\ldots+{ }_{n-1}^{n+1}\left[\frac{g_{j}}{g_{0}}\right]-{ }_{n}^{n+1}\right\},
$$

2. when $n$ is even

$$
\frac{-1}{g_{0}}\left\{\left[\frac{g_{j}(x)}{g_{0}}\right]^{n}-{ }_{1}^{n+1}\left[\frac{g_{j}(x)}{g_{0}}\right]^{n-1}+{ }_{2}^{n+1}\left[\frac{g_{j}}{g_{0}}\right]^{n-2}+\ldots+{ }_{n-1}^{n+1}\left[\frac{g_{j}}{g_{0}}\right]-{ }_{n}^{n+1}\right\},
$$

\subsection{OUTLINES OF STANDARD OPTIMIZATION ALGORITHM:}

In this algorithm we use the method of variable metric with cubic interpolation to compute the new point, and the problem is to find the minimum value to the non linear constrained function by using the BFGS formulae.

$\underline{\text { Step } 1}$ : take $x_{1}$ as initial point, $\mathrm{k}=1$.

Step 2: $\mathrm{H}=\mathrm{I}$.

Step 3: find the value of function at the point $x_{1}$, and put it in $f_{1}$.

Step 4: $d=-H g_{k}$.

Step 5: Use the cubic interpolation to find the step $\lambda$ to compute

$$
x_{k+1}=x_{k}+\lambda \cdot d_{k}
$$

Step 6: use the BFGS formulae to update H.

Step7: comparison while $\left|\left(f_{2}-f_{1}\right) / f_{1}\right| \prec t o l$ if this condition is satisfied, go to step 8 else put new $f$ instead of old $f$ and go to step 3 .

$\underline{\text { Step } 8}$ : compares while $r \prec$ tol stop else $r=r / 10$ and go to step 2 .

\subsection{FLOW CHART OF STANDARD ALGORITHM:}

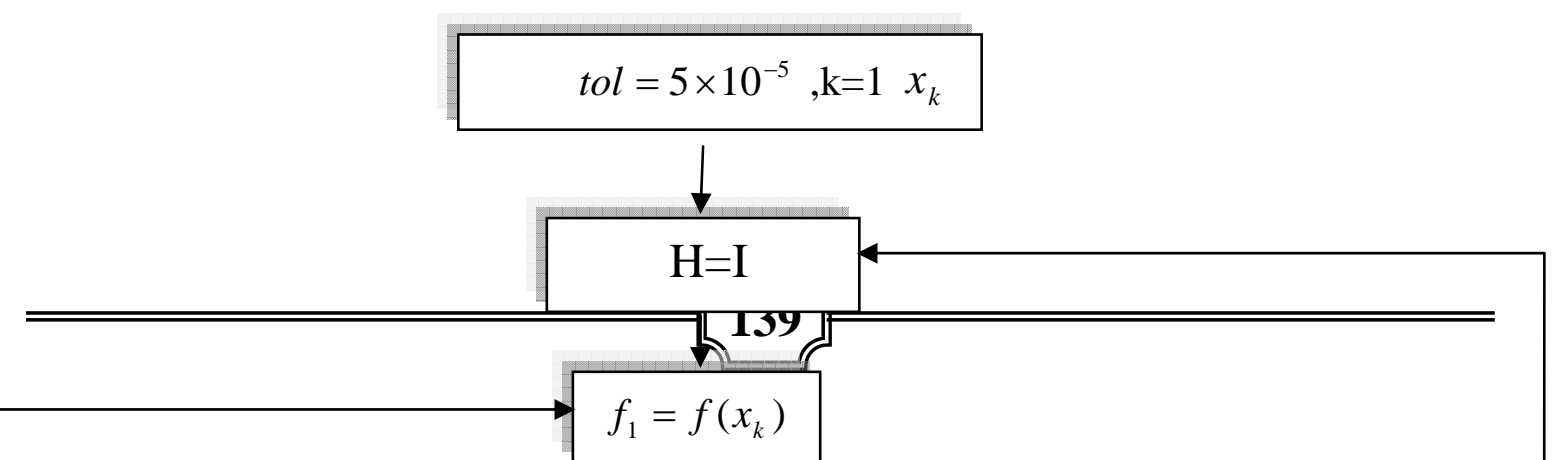




\section{A NEW HYBIRD BARRIER ALGORITHM:}

By using the new formula which is given in the equation (29), (30), and combine it with the standard algorithm we obtain the new hybrid algorithm and the outlines of this algorithm are as follow:

Step 1: take $x_{1}$ as initial point , $\mathrm{k}=1$. 
Step 2: find $z=f\left(x_{k}\right)$ and then find $r_{p}=|z| / \sum_{j=1}^{m} p_{j}(x)$.

Step 3: compute the sum of the constrained ( $\mathrm{z} 1, \mathrm{z} 2$ ) as follows :

$\forall j=1, \ldots m$, s.t $\mathrm{m}$ : sum of constrained If $g_{j}(x)>g_{0}$ compute $\mathrm{z} 1$

as one of the form (29), (30) and if $g_{j}(x) \leq g_{0}$ compute z2 as

equation (16), then find the sum $\mathrm{z} 3$ of the two cases.

Step 4: compute c as the equation (20).

Step 5: compute $g_{0}=-c\left(r_{p}\right)^{a}$.

Step 6: $\mathrm{H}=\mathrm{I}$.

Step 7: find the value of function at the point $x_{1}$, and put it in $f_{1}$.

Step 8: $d=-H g_{k}$.

Step 9: use the cubic interpolation to find the step $\lambda$ to compute $x_{k+1}=x_{k}+\lambda \cdot d_{k}$.

Step 10: use the BFGS formulae to update $\mathrm{H}$.

Step11: comparison while $\left|\left(f_{2}-f_{1}\right) / f_{1}\right| \prec t o l$ if this condition satisfy go to step 12 else put $f_{2}$ in place of $f_{1}$ and go to step 7 .

Step 12: compares if $r \prec t o l$ stop else $r=r / 10$ and go to step 6 .

\subsection{FLOW CHART OF THE NEW HYBIRD ALGORITHM:}

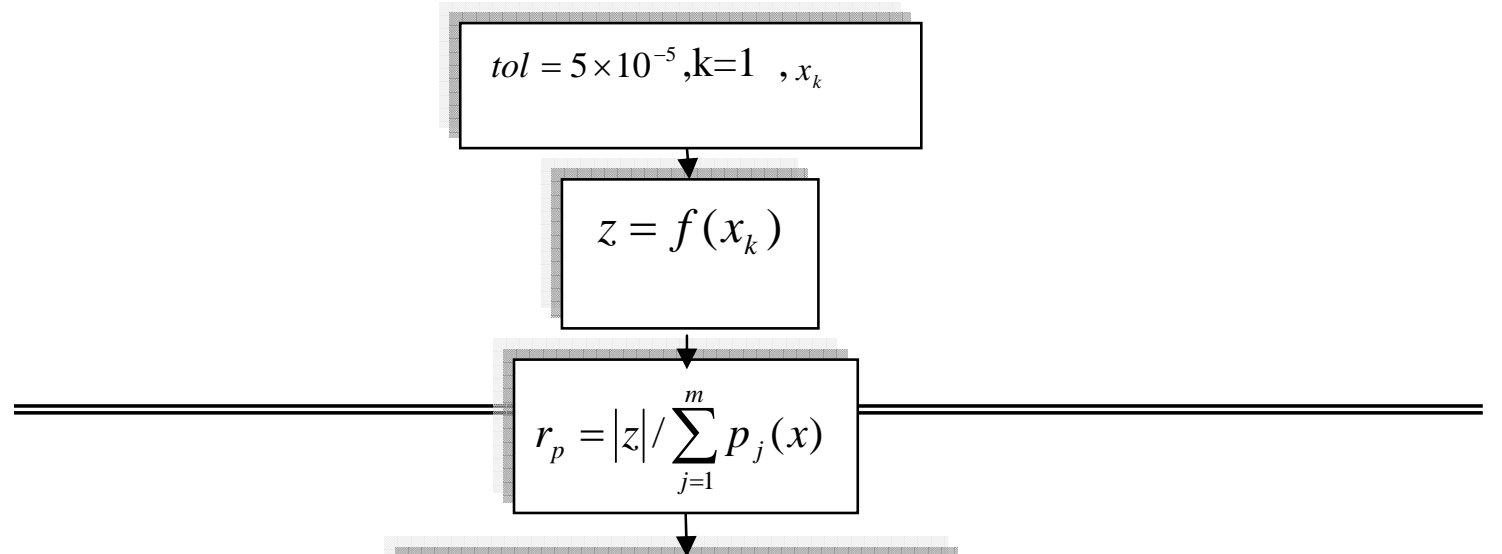




\subsection{Convergence of the new proposed algorithm;}

Consider only the barrier methods (penalty methods can be analyzed in a similar way) applied to the problem $\min f(x)$

s.t $\quad g_{i}(x) \geq 0 \quad, i=1, \ldots . m$

Let $S$ and $S_{0}$ denote, respectively, the feasible region and its interior, i.e. 


$$
\begin{aligned}
& S=\{x \mid g i(x) \geq 0, i=1, \ldots, m\}, \\
& S_{0}=\{x \mid g i(x)>0, i=1, . ., m\}
\end{aligned}
$$

\subsection{Assumptions:}

(1) $f$ and all $g i(i=1, \ldots, m)$ are continuous. $\phi$ is a continuous function on $S_{0}$, and $f(x) \rightarrow+\infty$ when $x$ approaches the boundary of $S$.

(2) For any $a$, the set $\{x \mid x \in S, f(x) \leq a\}$ is bounded.

(3) $S_{0}$ is not empty.

(4) Any $y \in S$ can be approached by a sequence $\left\{x_{k}\right\}$ in $S_{0}$ : $x_{k} \longrightarrow y \quad, x_{k} \in S^{0}$

\subsection{Theorem (Convergence of the Barrier Methods)}

Let $\lim _{k \rightarrow \infty} \mu_{k}=0 ; B(x, \mu)=f(x)+\mu \phi(x) ; \mu_{1} \geq \mu_{2} \geq \ldots \quad$ and $x_{k}$ is a global minimizer of problem $\min _{k \rightarrow \infty} B\left(x, \mu_{k}\right)$, then, for $k=1,2,$. .

(a) $f\left(x_{k+1}\right) \leq f\left(x_{k}\right)$

(b) $\phi\left(x_{k+1}\right) \geq \phi\left(x_{k}\right)$

(c) $\left\{x_{k}\right\}$ has a convergent subsequence.

(d) if the subsequence $\left\{x_{k} \mid k \in K\right\}$ concerges to $\hat{x^{\wedge}}$, then $\hat{x^{\wedge}}$ must be a global solution of the problem (31).

\section{Proof:}

(a) For each $k, x_{k}$ is the solution of $\min _{x \in S^{0}} B\left(x, \mu_{k}\right)$,

So,

$B\left(x_{k}, \mu_{k}\right) \leq B\left(x_{k+1}, \mu_{k}\right) \Leftrightarrow f\left(x_{k}\right)+\mu_{k} \phi\left(x_{k}\right) \leq f\left(x_{k+1}\right)+\mu_{k} \phi\left(x_{k+1}\right)$,

$B\left(x_{k+1}, \mu_{k+1}\right) \leq B\left(x_{k}, \mu_{k+1}\right) \Leftrightarrow f\left(x_{k+1}\right)+\mu_{k+1} \phi\left(x_{k+1}\right) \leq f\left(x_{k}\right)+\mu_{k+1} \phi\left(x_{k}\right)$,

$\mu_{k+1} \times(35)+\mu_{k} \times(36)$,

$\Rightarrow \mu_{k+1} f\left(x_{k}\right)+\mu_{k} f\left(x_{k+1}\right) \leq \mu_{k+1} f\left(x_{k+1}\right)+\mu_{k} f\left(x_{k}\right)$

$\Rightarrow\left(\mu_{k}-\mu_{k+1}\right) f\left(x_{k+1}\right) \leq\left(\mu_{k}-\mu_{k+1}\right) f\left(x_{k}\right)$

$\Rightarrow\left(\mu_{k}-\mu_{k+1}\right)\left(f\left(x_{k}\right)-f\left(x_{k+1}\right) \geq 0\right.$

$\Rightarrow f\left(x_{k}\right) \geq f\left(x_{k+1}\right)$ where $\left(\mu_{k} \geq \mu_{k+1}\right)$,

(b) 
$\Rightarrow \mu_{k} \phi\left(x_{k}\right) \leq \mu_{k} \phi\left(x_{k+1}\right)$

$\Rightarrow \phi\left(x_{k}\right) \leq \phi\left(x_{k+1}\right)$

(c) Let $S^{\prime}=\left\{x \in S \mid f(x) \leq f\left(x_{1}\right)\right\}$ Since $f\left(x_{k+1}\right) \leq f\left(x_{k}\right) \leq \ldots \leq f\left(x_{1}\right)$

$\Rightarrow x_{k} \in S$

As

continuity of $f \Rightarrow S^{\prime}$ is a closed set; and assumption (2) $\Rightarrow S^{\prime}$ is a bounded set,

$\Rightarrow S^{\prime}$ is a compact set.

So, $\left\{x_{k}\right\}$ must have a convergent subsequence.

(d) Let $x_{k} \stackrel{k}{\longrightarrow} \hat{x}$ we need to prove that $\hat{x^{\wedge}}$ must be an optimal solution of problem (31).

(i) Since $x_{k} \in S^{0}, g_{i}\left(x_{k}\right)>0 \quad(\forall k, \forall i) \quad$ Then, as $K \stackrel{k}{\longrightarrow} \infty, \quad g_{i}(\hat{x}) \geq 0$, $\forall i=1, \ldots m . \Rightarrow \hat{x} \in S$, i.e. $\hat{x^{\prime}}$ is a feasible solution of problem (31).

(ii) Let $x^{*}$ be a global minimizer of problem (31). By assumption (4), and the continuity of $f$, for any $\varepsilon>0$, there exists $x_{\varepsilon} \in S$ such that $f\left(x_{\varepsilon}\right)<f\left(x_{*}\right)+\varepsilon$.

Due to (31), $B\left(x_{k}, \mu_{k}\right) \leq B\left(x_{\varepsilon}, \mu_{k}\right)$ i.e $f\left(x_{k}\right)+\mu_{k} \phi\left(x_{k}\right) \leq f\left(x_{k}\right)+\mu_{k} \phi\left(x_{\varepsilon}\right)<f\left(x_{*}\right)+\varepsilon+\mu_{k} \phi\left(x_{\varepsilon}\right)$,

If $\hat{x} \in S^{0}$, then $\phi(\hat{x})$ is a finite number, and $\phi\left(x_{k}\right) \stackrel{k}{\longrightarrow} \phi(\hat{x})$

Let $k \stackrel{k}{\longrightarrow} \infty$ from (36),

$f(\hat{x})+0 . \phi(\hat{x}) \leq f\left(x_{*}\right)+\varepsilon+0 . \phi\left(x_{\varepsilon}\right)$ i.e

$f(\hat{x}) \leq f\left(x_{*}\right)+\varepsilon$,

If $\hat{x} \notin S^{0}$, i.e. $\hat{x}$ is on the boundary of $S$, then $\phi(\hat{x})=+\infty$ So, for large $k$ $\in K, \varphi\left(x_{k}\right)>0$ we have from (38), $f\left(x_{k}\right) \leq f\left(x_{k}\right)+\mu_{k} \phi\left(x_{\varepsilon}\right)<f\left(x_{*}\right)+\varepsilon+\mu_{k} \phi\left(x_{\varepsilon}\right)$, for large $k \in K$.

Let $k \stackrel{k}{\longrightarrow} \infty$ from the above inequalities, we have: $f(\hat{x}) \leq f\left(x_{*}\right)+\varepsilon$,

From (39.1) and (39.2), for any $\varepsilon>0, \quad f(\hat{x}) \leq f\left(x_{*}\right)+\varepsilon$ 
$\Rightarrow f(\hat{x}) \leq f\left(x_{*}\right)$

$\Rightarrow f(\hat{x})=f\left(x_{*}\right)$

$\Rightarrow x^{\wedge}$ is a global minimizer of problem (31).

\section{NUMARICAL RESULTS:}

Test of comparison contains (8) functions given in the appendix. All results obtained by using double precision by Pentium Computer and are programmed by Matlab language .

The efficiency of this algorithm depend on the number of function evaluation and number of iteration, and results are explained in tables (5.1) , (5.2) .

The line search which is used in this algorithm is the cubic interpolation and the objective function and constraint are given for each test problem.

The stopping criteria which is used in all algorithms for all cases is $r<5 \times 10^{-5}$.

Our numerical results shows that the new hybrid algorithm is effected when the comparison depend on NOI and NOF.

Table (5.1): Comparison between standard and hybrid algorithm.

\begin{tabular}{|c|c|c|c|c|}
\hline \multirow{2}{*}{$\begin{array}{c}\text { Test } \\
\text { function }\end{array}$} & \multicolumn{2}{|c|}{ Standard } & \multicolumn{2}{c|}{ Hybrid } \\
\cline { 2 - 5 } & NOI & NOF & NOI & NOF \\
\hline 1 & 11 & 43 & 20 & 107 \\
\hline \hline 2 & $\begin{array}{c}\text { Fail to } \\
\text { converge }\end{array}$ & 4 & 19 \\
\hline
\end{tabular}




\begin{tabular}{|c||c||c|c|c||}
\hline 3 & 11 & 50 & 15 & 78 \\
\hline \hline 4 & $\begin{array}{c}\text { Fail to } \\
\text { converge }\end{array}$ & 16 & 80 \\
\hline \hline 5 & $\begin{array}{r}\text { Fail to } \\
\text { converge }\end{array}$ & 17 & 102 \\
\hline \hline 6 & 9 & 57 & 12 & 77 \\
\hline \hline 7 & 17 & 70 & 14 & 73 \\
\hline \hline 8 & 13 & 55 & 11 & 75 \\
\hline \hline
\end{tabular}

(5.1)

Table (5.2): if the standard algorithm take $100 \%$ of NOI,NOF

\begin{tabular}{|c|c|c|}
\hline Tools & NOI & NOF \\
\hline \hline standard & $100 \%$ & $\% 100$ \\
\hline Hybrid & $\% 62$ & $\% 37$ \\
\hline
\end{tabular}

$(5.2)$

\section{Appendix}

1.

$$
\begin{aligned}
& \min f(x)=\left(x_{1}-3\right)^{2}+\left(x_{2}-4\right)^{2} \\
& \text { s.t. } \quad 2 x_{1}^{2}-x_{2}^{2}+34 \geq 0 \\
& \quad 2 x_{1}-3 x_{2}+18 \geq 0
\end{aligned}
$$

2. 
$\min f(x)=-x_{1} x_{2} x_{3}$

s.t. $2 x_{1}^{2}+x_{2}^{2}+3 x_{3}^{2}-51 \geq 0$

3.

$$
\begin{array}{ll}
\min & f(x)=x_{1}{ }^{2}+x_{2}{ }^{2} \\
\text { s.t. } & x_{1}+1 \geq 0 \\
& x_{2}+1 \geq 0
\end{array}
$$

4.

$$
\begin{array}{ll}
\min & f(x)=-x_{1}^{2}-x_{2}^{2} \\
\text { s.t. } & x_{1}^{2}+x_{2}^{2}-17 x_{1}-5_{2}+66 \geq 0 \\
& x_{1}^{2}+x_{2}^{2}-10 x_{1}-10 x_{2}+41 \geq 0 \\
& x_{1}^{2}+x_{2}^{2}-4 x_{1}-14 x_{2}+45 \geq 0 \\
& x_{1}+7 \geq 0 \\
& x_{2}+7 \geq 0
\end{array}
$$

5.

$\min f(x)=\left(x_{1}-x_{2}\right)^{2}+\left(x_{1}-2 x_{2}\right)^{2}$

s.t. $-x_{1}^{2}-x_{2} \leq 0$

6.

$\min f(x)=x_{1}{ }^{2}+3 x_{2}{ }^{2}+1.5 x_{3}^{2}$

s.t. $2 x_{1}+x_{2}+x_{3}-20 \leq 0$

$$
x_{1}+x_{3}-10 \leq 0
$$

7.

$$
\begin{array}{ll}
\min & f(x)=2 x_{1}^{2}+2 x_{1} x_{2}+x_{2}{ }^{2}-10 x_{1}-10 x_{2} \\
\text { s.t. } & x_{1}^{2}+x_{2}^{2}+5 \geq 0 \\
& 3 x_{1}-x_{2}+6 \geq 0
\end{array}
$$

8.

$$
\begin{array}{ll}
\min & f(x)=x_{1}{ }^{2}+x_{2}{ }^{2} \\
\text { s.t. } & x^{2}{ }_{1}+x_{2}{ }^{2} \leq 9 \\
& x_{1}+x_{2} \leq 1
\end{array}
$$

\section{References:}

1. Carrol, C. W., "The created Response Surface Technique for Optimization Non Linear Restrained Systems", Op.Res, pp .169184, 1961. 
2. Fiaco. A, ormick G. Mc,. ,"NonLinear Programming Sequential Unconstrained Minimization Techniques,", SIAM classic in Applied Mathematics, SIAM Philadelphia, PA, 1990.

3. Frich, K. R., "The logarithmic Potential Method of Convex Programming Memorandum", University Institute of Economics Oslo. 1955.

4. Toint, ph. L and Ncholas L. M. " A note On The Second Order Convergence of Optimization Algorithm by Using Barrier Function" A current survey, Report au/1, IBMJ.J. Waston Research Center, U.S.A., 1997.

\section{Internet References:}

5. AOE/ESM 4084 “Engineering Design Optimization”. Indirect or Transformation Methods (or SUMT Techniques). for n-Dimensional Constrained Minimization, http://www.esm.vt.edu/ zgurdal/COURSES/4084/4084Docs/LECTURES/15.pdf

6. "Penalty and Barrier Methods", 2001,http://www.cityu.edu.uk / ma / staff / zhang / MA66-11-16 .pdf .

7. "Multidisciplinary System Design Optimization" (MSDO), 2004, Karen willcox. http://ocw.mit.edu/.../16-888 Spring-2004 /F1388ED1-61E8-4513-AFBC-FFBFD8FD 4654/0/17_numopt2.pdf

8. "Penalty and Barrier Methods for Constraiend Optimization", 2004. http://ocw. Mit. edu / NR /rdonlyres/Sloan-Shool-ofManagement / 15-084 Jspring 2004 / A8E10BC*6B04 - 4D64-94 F2 - FB697408 B1 FF / 0 / lec 10 - Penalty -mt.pdf

9. Kevin Ross "Optimization of Non Linear Objectives,with NonLinear Constraints", 2005.http:// soe. ucsc. edu / classes / ism 206 / spring05 / lecture 20\% pritam 20\% Roy 20\% April 26 lecture. pdf. 